\section{Alphacypermethrin residues in milk following a pour-on treatment in buffaloes}

\author{
Giorgio Smaldone, Raffaele Marrone, \\ Claudia Chirollo, Lucia Vollano, Raffaella \\ Mercogliano, Aniello Anastasio \\ Dipartimento di Medicina Veterinaria e \\ Produzioni Animali, Università degli \\ Studi di Napoli Federico II, Napoli, Italy
}

\section{Abstract}

Aim of this study was to detect and quantify the residues of alphacypermethrin (ACYP), a pyrethroid insecticide, in buffalo milk. Ten animals were treated pour-on at the manufacturer's recommended cattle dose. Milk samples were collected from each animal before treatment, every $12 \mathrm{~h}$ for the first three days (12, $24,36,48,60,72 \mathrm{~h}$ ) and once on day 5 after treatment. Milk samples were subsequently analysed by ultraperformance liquid chromatography coupled with tandem mass spectrometry (UPLC-MS/MS). All samples showed levels of ACYP above the maximum residual limit of $20 \mathrm{ppb}$ established for bovine milk.

\section{Introduzione}

La specie bufalina si caratterizza per una spiccata capacità di adattamento all'ambiente. L'elevata resistenza agli ecto ed endoparassiti e la capacità di migliorare l'efficienza di utilizzazione degli alimenti, in condizioni di carenza foraggiera, hanno fatto sì che in passato, grazie all'allevamento di questa specie animale, si potesse creare un'economia in territori paludosi caratterizzati da produzioni foraggiere grossolane e dove ogni altro tipo di allevamento risultava impossibile. Nell'ultimo decennio l'allevamento bufalino ha subito un notevole incremento a livello mondiale e ancor più in Italia, che, con i suoi circa 370.000 mila capi, detiene il primato in Europa (banca dati nazionale - IZS Teramo). La popolazione bufalina mondiale è oggi stimata pari a oltre 190 milioni di capi. Negli ultimi 10 anni la popolazione mondiale è aumentata circa del 12,5\%, ma nonostante questi dati, l'allevamento bufalino, ad eccezione di alcune zone, viene considerato una realtà marginale rispetto a quello bovino e anche per questo le aziende farmaceutiche, nella messa a punto di nuove molecole farmacologiche, investono maggiormente nel comparto della zootecnia bovina o comunque in specie diverse da quella bufalina. Per alcune molecole ad azione antiparassitaria (in particolare per l'ivermectina) è stato dimostrato che la cinetica residuale è diversa tra le specie bovina e bufalina (Anastasio et al., 2002). Esiste pertanto un giustificato dubbio che eventuali limiti massimi residuali [maximum residue level (MRL)] di farmaci, stabiliti dagli organi di competenza a livello comunitario per la specie bovina, potrebbero non essere validi per la specie bufalina, con evidente rischio per il consumatore. Nell'ambito delle diverse molecole farmacologiche utilizzate nell'allevamento bufalino, un posto rilevante è occupato da quelle che svolgono azione antiparassitaria per le problematiche relative agli ectoparassiti. L'alfacipermetrina (ACYP) è un insetticida appartenente al gruppo dei piretroidi di sintesi, sostanze chimiche simili nella struttura alle piretrine naturali, classificati come moderatamente nocivi (classe II) dall'Organizzazione Mondiale della Sanità. È stato dimostrato che l'ACYP, disponibile in commercio dalla metà degli anni Ottanta, ritarda la chiusura dei canali di membrana nei neuroni sodio voltaggio-sensibili, inibisce i recettori acido -amminobutirrico (GABA) (Bloomquist, 1996) ed è efficace contro un'ampia gamma di parassiti In Italia, l'ACYP è commercializzata per la specie bovina come formulazione pour-on, con tempo zero di sospensione nel latte, per il trattamento di infestazioni da pidocchi. In relazione alla esiguità di studi relativi al grado di escrezione delle molecole nel latte di bufala $\mathrm{e}$ alla mancanza di informazioni sui fattori di trasferimento dei residui nei prodotti di trasformazione, scopo di questo lavoro è stato quello di valutare i livelli di ACYP in latte e mozzarella, dopo somministrazione pour-on a bufale.

\section{Materiali e Metodi}

Per la realizzazione di questo esperimento sono state utilizzate 10 bufale in lattazione (Bubalus bubalis) del peso medio di $500 \mathrm{~kg}$, con una produzione giornaliera di $9,4 \pm 2,07 \mathrm{~kg}$ di latte, mantenute al coperto durante tutto il periodo della sperimentazione. Le bufale selezionate non sono state trattate con nessuna sostanza ad azione farmacologica nei quattro mesi precedenti la prova. Al giorno 0 , prima di effettuare il trattamento, è stato prelevato un campione di latte e, successivamente, sulla cute asciutta, con un dosatore è stata applicata, sulla linea mediana dorsale dal garrese fino all'attaccatura della coda, una soluzione di ACYP all' $1,5 \%$ pour-on reperita in commercio alla dose di $0,000015 \mathrm{mg} / \mathrm{kg}$ indicata dal produttore. Per ogni animale il dosaggio è stato calcolato sulla base del rispettivo peso corporeo. Tutti i soggetti sono stati monitorati per un periodo di $4 \mathrm{~h}$ dopo il trattamento, per notare eventuali effetti collaterali, e, successiva-
Correspondence: Giorgio Smaldone, Dipartimento di Medicina Veterinaria e Produzioni Animali, Università degli Studi di Napoli Federico II, via Delpino 1, 80137 Napoli, Italy.

Tel. +39.081.2536464 - Fax: +39.081 .458683 .

E-mail: giorgio.smaldone@unina.it

Key words: Alphacypermethrin, Buffaloes, Milk, Pour-on treatment.

Received for publication: 15 January 2013.

Revision received: 15 April 2013.

Accepted for publication: 15 April 2013.

This work is licensed under a Creative Commons Attribution 3.0 License (by-nc 3.0).

(C) Copyright G. Smaldone et al., 2013

Licensee PAGEPress, Italy

Italian Journal of Food Safety 2013; 2:e19

doi:10.4081/ijfs.2013.e19

mente, ogni giorno per tutta la durata della sperimentazione. Anche il sito di applicazione è stato esaminato per evidenziare eventuali modificazione del pelo o della cute.

Da ciascun animale sono stati raccolti campioni di latte a $12,24,36,48,60,72,120 \mathrm{~h}$ dal trattamento.

Tutti i campioni sono stati congelati nel più breve tempo possibile a $-80^{\circ} \mathrm{C}$, in attesa delle analisi che sono state effettuate presso il Food Safety Department of Food Research Centre, Teagasc di Dublino. Sono stati utilizzati gli standard analitici dell'ACYP (99,7\%) (Sigma Aldrich, St. Louis, M0, USA) e standard interni di deltametrina (99,8\%) (Honeywell Riedel-de Haen, Seelze, Germania). L'acqua ultra pura $(18.2 \mathrm{M} \Omega)$ è stata ottenuta utilizzando il sistema di depurazione Milli-Q Plus (Millipore, Billerica, MA, USA). Tutti i reagenti usati erano high performance liquid chromatography (HPLC) grade.

È stata preparata una soluzione madre di ACYP con acetonitrile di $1 \mathrm{mg} \mathrm{mL}^{-1}$ e da questa è stata ottenuta la soluzione standard di lavoro di ACYP di $1 \mathrm{~g} \mathrm{~mL}^{-1}$ in acetonitrile. La soluzione madre dello standard interno di deltametrina è stata di $2 \mathrm{mg} \mathrm{mL}^{-1}$; quella di lavoro di $2 \mathrm{~g}$ $\mathrm{mL}^{-1}$ in acetonitrile. Tutte le soluzioni standard sono state stoccate $\mathrm{a}+6^{\circ} \mathrm{C}$.

Le curve di calibrazione sono state preparate fortificando campioni di latte negativo con concentrazioni di 1, 2, 5, 10, 20 e $40 \mathrm{~g} \mathrm{~kg}^{-1}$. Il recupero è stato misurato analizzando i picchi estratti dai campioni di latte fortificati con la soluzione di ACYP a concentrazioni di 5 e 20 $\mathrm{mg} \mathrm{kg}{ }^{-1}$. Per saggiare l'accuratezza del metodo, campioni di latte di animali non trattati sono stati fortificati con soluzioni standard e successivamente estratti, purificati ed analizzati come sopra descritto. La precisione del metodo 
è stata determinata analizzando campioni spiked con soluzioni standard delle due molecole. La specificità è stata confermata dall'analisi di campioni bianchi (controllo) di animali non trattati. Il limite di decisione (CC), cioè il limite al quale e oltre il quale è possibile concludere con una probabilità di errore pari ad che un campione è non conforme e la capacità di rilevazione (CC), che rappresenta il contenuto più piccolo della sostanza che è possibile rilevare, identificare e/o quantificare in un campione con una probabilità di errore di , calcolati secondo le linee guida internazionali (Commissione Europea, 2002) sono stati rispettivamente 0,62165 e 1,05921.

Al momento delle analisi i campioni di latte sono stati scongelati e pesati, dopo essere stati lievemente centrifugati a temperatura ambiente per favorirne l'omogeneizzazione, e $10 \mathrm{~g}$ del campione sono stati fortificati con $100 \mathrm{~L} \mathrm{di}$ soluzione di lavoro dello standard interno $(2 \mathrm{~g}$ $\mathrm{mL}^{-1}$ ) ed i bianchi sono stati fortificati con la soluzione standard di ACYP ( $\left.1 \mathrm{~g} \mathrm{~mL}^{-1}\right)$ al fine di avere concentrazioni di $1,2,5,10,20$ e 40 ppb nel latte di bufala. L'estrazione è stata eseguita aggiungendo acetonitrile $(10 \mathrm{~mL})$, solfato di magnesio (4 g) e cloruro di sodio (1 g) per ogni campione e agitando vigorosamente per 1 min. Successivamente i campioni sono stati centrifugati $(2842 \times g, 10 \mathrm{~min})$ e l'estratto filtrato attraverso filtri di 0,2 micron in vials da 200 L. La separazione è stata effettuata mediante un sistema UHPLC-MS/MS. È stata utilizzata una colonna analitica Acquity UHPLC ${ }^{\circledR}$ $\mathrm{BEH}_{8}(50 \mathrm{mmx} 2,1 \mathrm{~mm}, 1,7 \mu \mathrm{m})$ (Waters Corp., Milford, MA, USA) con una precolonna Vanguard $\mathrm{C}_{8}(5 \mathrm{mmx} 2,1 \mathrm{~mm})$ riscaldata a $60^{\circ} \mathrm{C}$. È stata eseguita una separazione a gradiente binario con flusso a velocità di $0,5 \mathrm{~mL}$ $\mathrm{min}^{-1}$. La fase mobile A consisteva di una soluzione acquosa di ammonio acetato $(2 \mathrm{mM})$ mentre la fase mobile B era costituita da metanolo $(\mathrm{MeOH})$. Il profilo del gradiente è stato il seguente:

$\begin{array}{ll}\text { A. } & 0 \rightarrow 0,1 \min , 50 \% \mathrm{~B} ; \\ \text { B. } & 3 \min , 95 \% \mathrm{~B} ; \\ \text { C. } & 3 \rightarrow 4 \min , 95 \% \mathrm{~B} \\ \text { D. } & 4,01 \min , 50 \% \mathrm{~B} ; \\ \text { E. } & 4,01 \rightarrow 5 \min , 50 \% \mathrm{~B} \text {; }\end{array}$

In totale la corsa cromatografica è durata 6 min. Il lavaggio dell'UHPLC è effettuato con una fase mobile debole di $\mathrm{H}_{2} \mathrm{O}: \mathrm{MeOH}$ (90:10 $\mathrm{v} / \mathrm{v})$ ed una più energica con $\mathrm{MeOH}: \mathrm{H}_{2} \mathrm{O}(90: 10$ $\mathrm{v} / \mathrm{v})$. Il volume dell'iniezione del campione era di 5 L. L'ACYP è stata individuata utilizzando un sistema a triplo quadropolo equipaggiato con una sonda electrospray che opera in modalità di ionizzazione positiva (Waters Corp.). L'azoto è stato utilizzato per la nebulizzazione, la desolvatazione (1100 $\mathrm{L} \mathrm{hr}^{-1}$ ) e come cono di gas ( $\left.200 \mathrm{hr} \mathrm{L}^{-1}\right)$. Le temperature iniziale e di desolvatazione erano rispettivamente 140 e $400^{\circ} \mathrm{C}$. Si è utilizzato un voltaggio capillare di $3.0 \mathrm{kV}$. L'argon è stato usato come gas di collisione. Il sistema UHPLC-MS/MS (Waters Acquity UHPLC system; Waters Corp.) è stato controllato dal software MassLynxTM e l'elaborazione dei dati è stata sviluppata dal software TargetLynxTM (Waters Corp.). Lo schema del settaggio cromatografico per la determinazione dei residui di ACYP è riportato nella Tabella 1.

\section{Risultati}

Le concentrazioni di ACYP nel latte rinvenute nei 10 soggetti ai diversi intervalli di tempo sono riportate nella Figura 1.
Da esso si evince che la massima concentrazione media di 34,5 ppb è stata raggiunta dopo $24 \mathrm{~h}$. I livelli sono poi diminuiti gradualmente e sono risultati al di sotto del limite di rilevabilità del metodo alla $120^{\mathrm{a}}$ ora post-trattamento. Fino al controllo effettuato $24 \mathrm{~h}$ dopo il trattamento il livello medio di ACYP è stato superiore al MRL fissato per il latte bovino (20 ppb) anche se in n. 2 soggetti concentrazioni superiori a $20 \mathrm{ppb}$ sono state osservate fino alla $48^{\mathrm{a}}$ ora.

\section{Discussione}

I risultati confermano la presenza di residui di ACYP nel latte di bufala, in analogia a quanto riscontrato per il latte bovino (Bissacot $\mathrm{e}$ Vassilieff, 1997a, 1997b), per il quale non è previsto alcun tempo di sospensione dopo trattamento delle bovine. Uno studio sulla stabilità durante lo stoccaggio della cipermetrina $\mathrm{e}$ dei suoi residui e metaboliti nel latte bovino e nei tessuti (muscolo, fegato e grasso) è stato condotto in precedenza nel 1995 da Barrett e Pearsall. I risultati indicano che la cipermetrina ed i suoi metaboliti sono stabili nel muscolo bovino e nel grasso per almeno 12 mesi e nel fegato e nel latte per almeno 3 mesi se stocca-

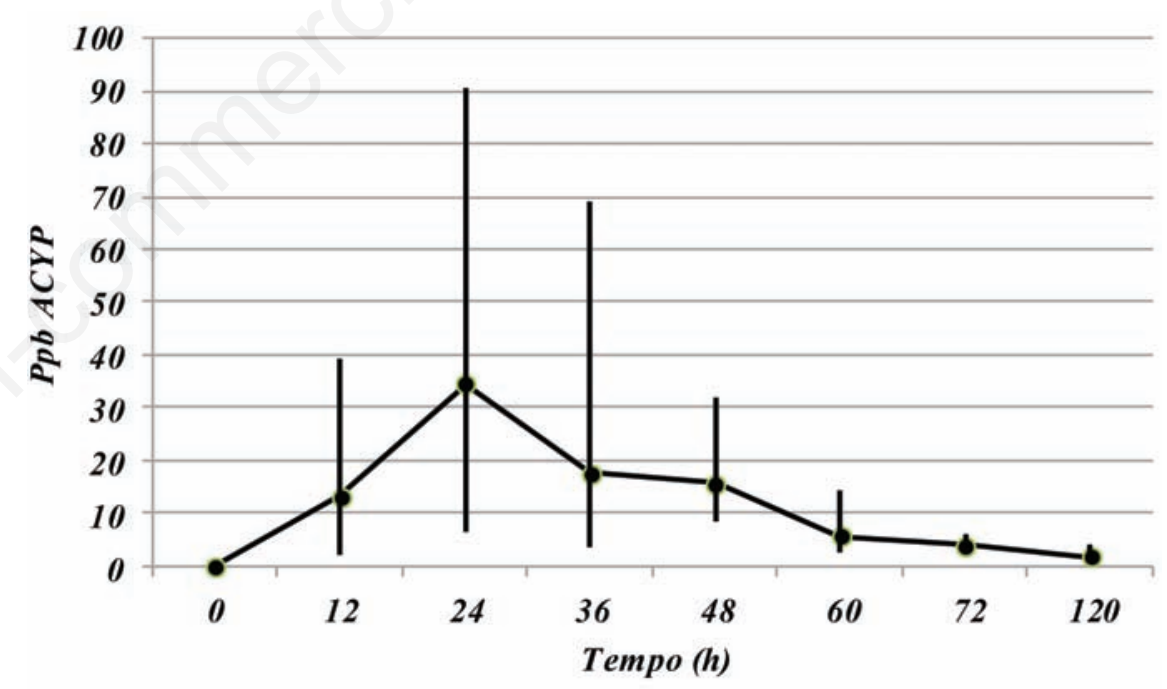

Figura 1. Livelli (linea orizzontale $=$ media; linea verticale $=$ minimo-massimo $)$ di alfacipermetrina in 10 campioni di latte di bufale trattate pour-on.

Tabella 1. Schema del settaggio cromatografico per la determinazione dei residui di alfacipermetrina.

\begin{tabular}{|c|c|c|c|c|c|c|}
\hline $\begin{array}{l}\text { Obiettivo sostanza e } \\
\text { standard interno relativo }\end{array}$ & $\begin{array}{l}\text { Ione iniziale } \\
(\mathrm{m} / \mathrm{z})\end{array}$ & $\begin{array}{l}\text { Ione finale } \\
\qquad(\mathrm{m} / \mathrm{z})\end{array}$ & $\begin{array}{l}\text { Tempo di sosta } \\
\text { (s) }\end{array}$ & $\begin{array}{l}\text { Voltaggio di cono } \\
\text { (V) }\end{array}$ & $\begin{array}{l}\text { Energia di collisione } \\
\qquad(\mathrm{eV})\end{array}$ & $\begin{array}{l}\text { Tempo di ritenzione } \\
\text { (min) }\end{array}$ \\
\hline ACYP & $433,0 \pm 0,5$ & $\begin{array}{l}190,80 \pm 0,5 \\
415,90 \pm 0,5\end{array}$ & $\begin{array}{l}0,035 \\
0,035\end{array}$ & $\begin{array}{l}20 \\
20\end{array}$ & $\begin{array}{c}14 \\
9\end{array}$ & $\begin{array}{l}2,51 \\
2,51\end{array}$ \\
\hline Standard interno del deltametrina & a $523,05 \pm 0,5$ & $280,9 \pm 0,5$ & 0,035 & 17 & 15 & 2,51 \\
\hline
\end{tabular}

ACYP, alfacipermetrina. 
ti in congelatore.

Le concentrazioni medie di ACYP rinvenute nel latte di bufale trattate con la dose indicata per il bovino superano, fino a $24 \mathrm{~h}$ post-trattamento, il MRL previsto per il latte bovino. La concentrazione del farmaco alla $36^{\text {a }}$ ora è risultata superiore a quella rinvenuta in vacche da latte $(13 \mathrm{ppb})$ alla $28^{\mathrm{a}}$ ora in seguito ad una somministrazione del farmaco, per via orale e per 28 giorni consecutivi, di $5 \mathrm{mg}$ di principio attivo per chilo di razione giornaliera (Audrey et al., 1997).

I livelli più alti, rispetto al bovino, riscontrati fino alla $36^{\text {a }}$ ora nel latte di bufala potrebbero anche indicare una diversa biodisponibilità della specie bufalina ed essere attribuibili a svariate cause, tra le quali la maggior quantità di grasso sottocutaneo (Bogan et al., 1988). Questa ipotesi sarebbe supportata da studi di analisi residuali in vari tessuti di bovine da latte trattate con cipermetrina per via orale, nelle quali il farmaco è stato ritrovato in campioni di crema e nel grasso peritoneale (Audrey et al., 1997), mentre non era stato rilevato nel latte durante l'intero periodo di somministrazione.

\section{Conclusioni}

Le concentrazioni di ACYP rinvenute nel latte di bufala destano preoccupazione e richiedono particolare attenzione dal momento che il latte di questa specie è quasi interamente destinato alla produzione di prodotti lattiero-caseari, mozzarella in particolare. Una corretta valutazione del rischio derivante dall'utilizzo di sostanze farmacologicamente attive in animali produttori di derrate alimentari, e connesso al consumo di tali derrate, deve necessariamente tenere in considerazione ulteriori fattori, non ultimo l'effettivo consumo dell'alimento.

La valutazione dell'efficacia antiparassitaria di una molecola, che nel caso specifico ha fornito risultati pienamente positivi nel corso di uno studio condotto parallelamente a questa sperimentazione, non può pertanto prescindere dalla verifica della presenza di residui in tutti gli alimenti destinati al consumo alimentare umano ottenibili dagli animali trattati.

La maggior parte dei farmaci è tuttavia autorizzata per l'utilizzo nella specie bovina e, in minor misura, in quella ovicaprina. I risultati dello studio sottolineano la necessità di ulteriori ricerche su cinetica ed escrezione dei farmaci nella specie bufalina, che presenta un metabolismo del tutto peculiare, molte volte diverso anche da specie affini, quale quella bovina.

\section{Bibliografia}

Anastasio A, Esposito M, Amorena M, Catellani P, Serpe L, Cortesi ML, 2002. Residue study of ivermectin in plasma, milk, and mozzarella cheese following subcutaneous administration to buffalo (Bubalus bubalis). J Agr Food Chem 50:5241-5.

Audrey W, Chen J, Fink M, Letinski DJ, Barrett GP, Jill C, 1997. pearsall residue of cypermethrin and its major acid metabolites in milk and tissues from dairy bovines treated with cypermethrin. J Agr Food Chem 45:4850-5.

Bissacot DZ, Vassilieff I, 1997a. HPLC determination of flumethrin, deltamethrin, cypermethrin, and cyhalothrin residues in the milk and blood of lactating dairy cows. $\mathrm{J}$ Anal Toxicol 21:397-402.

Bissacot DZ, Vassilieff I, 1997b. Pyrethroid residues in milk and blood of dairy cows following single topical applications. Vet Hum Toxicol 39:6-8.

Bloomquist JR, 1996. Ion channels as targets for insecticides. Annu Rev Entomol 41:16390.

Bogan JA, McKellar QA, 1988. The pharmacodynamics of ivermectin in sheep and cattle. J Vet Pharmacol Ther 11:260-8.

Commissione Europea, 2002. Decisione della commissione del 12 agosto 2002 che attua la direttiva 96/23/CE del Consiglio relativa al rendimento dei metodi analitici e all'interpretazione dei risultati, 2002/657/CE. In: Gazzetta Ufficiale, L 221/8, 17/08/2002. 